\title{
Macropinocytosis is responsible for the uptake of pathogenic and non-pathogenic mycobacteria by B lymphocytes (Raji cells)
}

Blanca Estela García-Pérez ${ }^{1}$, Juan José De la Cruz-López , Jorge Ismael Castañeda-Sánchez , Ana Rosa Muñóz-Duarte ${ }^{1}$, Alma Delia Hernández-Pérez² ${ }^{2}$ Hilda Villegas-Castrejón ${ }^{2}$, Ethel García-Latorre ${ }^{1}$ Angel Caamal-Ley ${ }^{1}$ and Julieta Luna-Herrera ${ }^{1 *}$

\begin{abstract}
Background: The classical roles of B cells include the production of antibodies and cytokines and the generation of immunological memory, these being key factors in the adaptive immune response. However, their role in innate immunity is currently being recognised. Traditionally, B cells have been considered non-phagocytic cells; therefore, the uptake of bacteria by B cells is not extensively documented. In this study, we analysed some of the features of non-specific bacterial uptake by B lymphocytes from the Raji cell line. In our model, B cells were infected with Mycobacterium tuberculosis (MTB), Mycobacterium smegmatis (MSM), and Salmonella typhimurium (ST).

Results: Our observations revealed that the Raji B cells were readily infected by the three bacteria that were studied. All of the infections induced changes in the cellular membrane during bacterial internalisation. $M$. smegmatis and S. typhimurium were able to induce important membrane changes that were characterised by abundant filopodia and lamellipodia formation. These membrane changes were driven by actin cytoskeletal rearrangements. The intracellular growth of these bacteria was also controlled by B cells. M. tuberculosis infection also induced actin rearrangement-driven membrane changes; however, the B cells were not able to control this infection. The phorbol 12-myristate 13-acetate (PMA) treatment of B cells induced filopodia and lamellipodia formation, the production of spacious vacuoles (macropinosomes), and the fluid-phase uptake that is characteristic of macropinocytosis. S. typhimurium infection induced the highest fluid-phase uptake, although both mycobacteria also induced fluid uptake. A macropinocytosis inhibitor such as amiloride was used and abolished the bacterial uptake and the fluid-phase uptake that is triggered during the bacterial infection.

Conclusions: Raji B cells can internalise S. typhimurium and mycobacteria through an active process, such as macropinocytosis, although the resolution of the infection depends on factors that are inherent in the virulence of each pathogen.
\end{abstract}

Keywords: Macropinocytosis, B lymphocytes, Raji cells, Mycobacterium smegmatis, Mycobacterium tuberculosis, Salmonella typhimurium, Cytoskeleton

\footnotetext{
* Correspondence: julietalunah@hotmail.com

'Deceased

'Immunology Department, Escuela Nacional de Ciencias Biológicas, Instituto Politécnico Nacional, Prolongación de Carpio y Plan de Ayala s/n, Z.P. 11340, México, D.F, México

Full list of author information is available at the end of the article
}

\section{Biomed Central}

(C) 2012 García-Pérez et al.; licensee BioMed Central Ltd. This is an Open Access article distributed under the terms of the Creative Commons Attribution License (http://creativecommons.org/licenses/by/2.0), which permits unrestricted use, distribution, and reproduction in any medium, provided the original work is properly cited. 


\section{Background}

B lymphocytes, in addition to their role as precursors of antibody producer cells (plasma cells), are responsible for the production of cytokines such as Interleukin (IL)4, IL-6, IL-10, and tumour necrosis factor-alpha (TNF$\alpha)$ [1] and act as antigen-presenting cells; consequently, these cells are essential in the adaptive immune response. Most antigen-presenting cells, such as macrophages and dendritic cells, take up antigens in bulk to sense the extracellular environment. B lymphocytes, however, recognise specific antigens in soluble or membrane-bound forms through the B-cell receptor (BCR) [2]. Upon BCR interaction with the antigen, a cascade of signal transduction and second messengers is generated and the antigen is internalised and subsequently processed for presentation through the major histocompatibility complex (MHC) II molecules for recognition by $\mathrm{T}$ cells [3]. The internalisation of the antigen in B cells occurs through at least two endocytic pathways: clathrin-mediated endocytosis and clathrin- and caveolin-independent endocytosis [3,4]. However, B cells also express a number of membrane receptors that initiate the innate immune response. These receptors include the Toll-like receptors (TLR) 1, 2 (low), 4 (low), and 6, 7, 9, and 10 [5]; low levels of DEC-205, which is a putative antigen uptake processing receptor [6]; the scavenger receptor Cluster of Differentiation 36 (CD36) [7]; and the Dendritic Cell-Specific Intracellular adhesive molecule-3-Grabbing Nonintegrin (DC-SIGN) [8]. Of these, DC-SIGN is present only after B-cell activation by CD40L and Interleukin (IL)-4, which makes the B-cell able to internalise Human Herpes virus 8 (HHV 8) $[8,9]$. In addition, CD36 enhances Toll-like receptor 2 (TLR2) signalling to induce cytokine production [7]. In contrast to the internalisation and procession of soluble antigen, the internalisation of particulate antigen by $\mathrm{B}$ cells has not been extensively investigated because, unlike "professional" phagocytes, B cells do not achieve phagocytosis [10]. However, recent evidence has shown that B cells can handle and process particle antigens, bacteria, and even protozoan parasites [10,11]. It has been demonstrated that the particulate presentation of a BCR-recognised soluble antigen enhances the adaptive response by up to $10^{5}$-fold [12]. In fact, the build-up of lipid-antigen in a particle can also be internalised by $\mathrm{B}$ cells and presented through their CD1d molecules to natural killer $\mathrm{T}$ (NKT) cells to induce a potent adaptive response, and this is only possible if the lipid particles are recognised by the BCR [13]. There are few studies on the uptake of bacteria by B cells. A number of bacteria, including mycobacteria [14], Salmonella typhimurium (ST) [15], IgM-opsonised Staphylococcus aureus [16], Listeria monocytogenes [17], and, more recently, Francisella tularensis [11], have been found to be internalised by B-cell lines or primary culture, although the precise mechanism that is responsible for their internalisation has not yet been elucidated. The B-cell bacterial endocytic activity has recently been recognised in lower-vertebrate species, such as fishes or frogs, and interestingly, these cells also exert potent antimicrobial activity [10]. We previously demonstrated that nonphagocytic cells, such as type II pneumocytes (A549 cells), internalised pathogenic and non-pathogenic mycobacteria through macropinocytosis $[18,19]$, and that this process was driven by metabolically active mycobacteria (live). To extend the study on the mycobacteriatriggered endocytic pathway that is responsible for the internalisation of invading non-phagocytic cells, we decided to analyse the internalisation of Mycobacterium tuberculosis (MTB) and Mycobacterium smegmatis (MSM) in B cells using scanning and transmission electron microscopy, confocal microscopy, and endocytic inhibitors to demonstrate that in Raji B cells, both of these mycobacteria are internalised through macropinocytosis. For validation, we compared our results with the internalisation features of Salmonella typhimurium, which was recently described to be internalised through macropinocytosis [20].

\section{Methods}

\section{B cells}

The Raji cell line, a human B lymphoblast cell line, was obtained from the American Type Culture Collection (ATCC, CCL-86). The cells were grown in RPMI-1640 with $10 \%$ fetal bovine serum (FBS) and antibiotics (25 $\mathrm{mg} / \mathrm{L}$ gentamicin and $50,000 \mathrm{U} / \mathrm{L}$ penicillin) at $37^{\circ} \mathrm{C}$ in an atmosphere with $5 \% \mathrm{CO}_{2}$.

\section{Bacteria and bacterial growth supernatants}

M. tuberculosis $\mathrm{H} 37 \mathrm{Rv}$ (ATCC) and M. smegmatis $\mathrm{mc}^{2}$ were grown in Middlebrook $7 \mathrm{H} 9$ broth, which was enriched with additional OADC for the growth of $M$. tuberculosis. Salmonella enterica serovar Typhimurium (Salmonella typhimurium, ST) (ATCC 14028) was grown in Luria broth. All bacteria were cultured at $37^{\circ} \mathrm{C}$ until achieving log-phase growth. Immediately prior to the use of the bacterial cultures in the different experiments, one aliquot of each culture was centrifuged at $10,000 \mathrm{rpm}$. The supernatant was then collected and all remaining bacteria were removed by filtration of the supernatant through $0.22-\mu \mathrm{m}$ filters; the bacteria-free supernatants were then maintained at $-70^{\circ} \mathrm{C}$ until use. The bacterial pellet was suspended in Hanks' balanced salt solution (HBSS) without phenol red and centrifuged; this washing step was repeated twice. The bacterial pellet was then resuspended in HBSS, adjusted to a McFarland number 1 tube, and diluted in RPMI-1640 medium 
with $1 \%$ FBS serum in the absence of antibiotics to reach the necessary bacteria-to-cell ratio.

\section{Survival of intracellular bacteria}

A suspension of B cells adjusted to a concentration of 2 $\times 10^{6}$ cells $/ \mathrm{mL}$ was prepared as described previously. The cells were infected with each bacterial suspension (M. tuberculosis, M. smegmatis, and S. typhimurium) and maintained at $37^{\circ} \mathrm{C}$ in a $\mathrm{CO}_{2}$ atmosphere. After $2 \mathrm{~h}$, the non-internalised bacteria were removed by low speed centrifugation (1,000 rpm for $5 \mathrm{~min})$, the supernatant was discarded, and the cells were suspended in HBSS. After this procedure was repeated three times, the cellular pellet was suspended in RPMI-1640 with $1 \%$ FBS, and $20 \mu \mathrm{g} / \mathrm{mL}$ of amikacin (Sigma); after two $\mathrm{h}$, the concentration of amikacin was decreased to $10 \mu \mathrm{g} / \mathrm{mL}$ to eliminate any extracellular bacteria; in the latter medium, the cells were incubated for $12,24,48$, and 72 $\mathrm{h}$ after infection with $M$. smegmatis and $M$. tuberculosis and for $6,12,18$, and $24 \mathrm{~h}$ after infection with S. typhimurium. After each time point, the cells were washed three times with HBSS using low-speed centrifugation $(1,000 \mathrm{rpm})$. To determine the number of intracellular bacteria, the washed cell pellet was lysed and resuspended in $500 \mu \mathrm{L}$ of sodium dodecyl sulphate (SDS) (0.25\%); after $3 \mathrm{~min}, 500 \mu \mathrm{L}$ of $5 \%$ bovine serum albumin (BSA) was added. The cell lysates were collected and maintained frozen at $-70^{\circ} \mathrm{C}$. To determine the colonyforming units (CFU), serial dilutions of the samples that were infected with $M$. tuberculosis and $M$. smegmatis were plated on Middlebrook 7H11 agar; similarly, the serial dilutions of the samples infected with S. typhimurium were plated on Luria agar.

\section{Bacterial and fluid-phase uptake by $B$ cells}

An aliquot of B cells in log-phase growth was centrifuged at 1,000 rpm and washed three times with HBSS. After the cell viability was determined using trypan blue dye, the suspension was adjusted to a concentration of $2 \times 10^{6}$ cells $/ \mathrm{mL}$ in RPMI-1640 with $1 \%$ FBS and $0.1 \mathrm{mg} / \mathrm{mL}$ dextran-FITC 70 (Sigma). The set of experiments on fluid-phase uptake were settled under the following conditions: (a) $1.0 \mu \mathrm{g} / \mathrm{mL}$ phorbol 12-myristate 13-acetate (PMA) (Sigma), (b) bacterial supernatant diluted by 1:10 in RPMI-1640, (c) M. smegmatis at a multiplicity of infection (MOI) of 10:1 and (d) M. tuberculosis at an MOI of 10:1, (e) S. typhimurium at an MOI of 20:1, and (f) control medium. In a 96-well sterile culture plate, a total of 200,000 treated cells were seeded in each well. The following procedure was followed for each condition: (1) quadruplicate samples were settled; (2) the plate was incubated at $37^{\circ} \mathrm{C}$ in a $\mathrm{CO}_{2}$ atmosphere; (3) after $15,60,90,120$, and $180 \mathrm{~min}$, the fluid-phase excess was removed by centrifugation; (4) the cells were washed three times with HBSS; and (5) the washed cells were resuspended in $100 \mu \mathrm{L}$ of HBSS. The fluorescence at each time point was measured as relative fluorescence units (RFU) using a plate fluorometer (Fluoroskan Ascent FL, Thermo) at a $485 \mathrm{~nm}$ excitation and a $538 \mathrm{~nm}$ emission. The inhibition of the fluid-phase uptake was analysed in the presence of several inhibitors, including (a) $3 \mu \mathrm{M}$ amiloride (AMIL), which is an ion exchange inhibitor that is used as an inhibitor of macropinocytosis [21,22], (b) $0.1 \mu \mathrm{M}$ wortmannin (WORT), a PI3K inhibitor [23] and (c) $3 \mu \mathrm{M}$ cytochalasin D (CD), a known inhibitor of actin polymerisation [24]. All of the inhibitors were purchased from Sigma. Each inhibitor was added to the respective cellular suspensions 30 min prior to treatment and was not removed during the experiment. The cells were processed as previously mentioned, and the resultant RFUs were recorded. The B-cell line viability in the presence of these inhibitors was monitored during the experiment. The cell viability was assessed by staining an aliquot with $0.2 \%$ trypan blue and calculating the percentage of cells that were not dyed. The viability in the control (no inhibitor) and treated cells reached $95 \%$. The fluid-phase uptake data were analysed for statistical significance using one-way analysis of variance (ANOVA) using the SigmaStat software. $P$ values $\leq$ 0.01 were considered statistically significant. The inhibition of the bacterial uptake was also analysed in the presence of amiloride using a protocol similar to that used in the previous experiments. Concentrations of 1,3 and 5 $\mathrm{mM}$ of amiloride were added to the cells $30 \mathrm{~min}$ prior to the addition of the bacteria; the inhibitor was maintained in the samples throughout the 90 min during which the bacterial uptake occurred. A set of untreated cells were infected with the same bacterial suspension for control. At the end of the incubation, the extracellular bacteria were removed by centrifugation, and the CFUs were determined as described previously. The cell viability was also assessed at the end of the experiment and was found to reach $>90 \%$ regardless of the concentration of inhibitor that was used.

\section{Transmission electron microscopy (TEM)}

Some of the features of the infection of B cells with $M$. tuberculosis, M. smegmatis, and S. typhimurium were analysed by TEM. Because PMA is known to act as a macropinocytosis inducer [25], the features of $B$ cells under PMA treatment were also analysed. B-cell suspensions were treated with $1.0 \mu \mathrm{g} / \mathrm{mL}$ of PMA for $1 \mathrm{~h}$ or infected for 1 and $24 \mathrm{~h}$ with the following bacterial suspensions: M. tuberculosis at an MOI of 10:1; M. smegmatis at an MOI of 10:1, and S. typhimurium at an MOI of 20:1. After treatment and infection, the suspension cells were washed four times by centrifugation at 1,000 rpm with PBS solution to remove any non-internalised bacteria and excess PMA. The cells were fixed with $2 \%$ 
glutaraldehyde solution in $0.1 \mathrm{M}$ PBS for $2 \mathrm{~h}$ at room temperature. The cells were then washed three times with PBS and post-fixed with osmium tetroxide for $1 \mathrm{~h}$ at $4^{\circ} \mathrm{C}$. The osmium tetroxide was removed by centrifugation with $\mathrm{PBS}$, and the pellet was processed following the standard procedure for TEM analysis [18]. The final sections obtained were examined under a transmission electron microscope (Philips, Tecnai 10, Holland).

\section{Scanning electron microscopy}

Fresh B-cell suspensions were prepared $\left(1 \times 10^{6}\right.$ cells/ $\mathrm{mL}$ ) and infected with non-labelled $M$. smegmatis, $M$. tuberculosis, or S. typhimurium for $1 \mathrm{~h}$ at $37^{\circ} \mathrm{C}$ and $5 \%$ $\mathrm{CO}_{2}$, according to the protocol described previously; in addition, some B-cell suspensions were treated with PMA instead of the bacterial cultures. The noninternalised bacteria or the excess PMA was removed by centrifugation using PBS, as described previously; the cell pellet was then fixed with $2 \%$ glutaraldehyde solution in PBS for $2 \mathrm{~h}$ at room temperature. The cells were then washed three times with PBS, post-fixed with osmium tetroxide for $1 \mathrm{~h}$ at $4^{\circ} \mathrm{C}$, and processed as previously described [18]. The cells were observed using a scanning electron microscope (Jeol-JSM-5800LV, Japan).

\section{Fluorescein isothiocyanate (FITC) bacterial staining}

To analyse the cytoskeletal rearrangements and bacterial intracellular localisation by confocal microscopy, the $M$. smegmatis, M. tuberculosis, and S. typhimurium bacteria were stained with Fluorescein isothiocyanate (FITC) (Sigma). The staining protocol included the following steps: (1) $1 \mathrm{~mL}$ of a McFarland number 3 bacterial suspension was washed by centrifugation, (2) the bacterial pellet was suspended in $1 \mathrm{~mL}$ of a phosphate buffered saline (PBS) solution (0.15 M, pH 7.2) that contained $0.1 \mathrm{mg} / \mathrm{mL}$ of FITC, and (3) the bacterial suspension was incubate for $30 \mathrm{~min}$ at $37^{\circ} \mathrm{C}$. The remaining dye was removed by centrifugation with PBS until the supernatant did not register any fluorescence when read on a plate fluorometer at a $485 \mathrm{~nm}$ excitation and a $538 \mathrm{~nm}$ emission (Fluoroskan Ascent FL, Thermo). The dyed bacterial pellet was adjusted to a McFarland number 1 tube in HBSS and then utilised in the respective experiments.

\section{Confocal microscopy}

A suspension of $\mathrm{B}$ cells at a concentration of $1 \times 10^{6}$ cells $/ \mathrm{mL}$ was processed as mentioned previously. The cells in suspension were infected for 1 and $3 \mathrm{~h}$ using a bacterial suspension of FITC-labelled M. tuberculosis, $M$. smegmatis, or S. typhimurium. The infections were performed at $37^{\circ} \mathrm{C}$ in an atmosphere with $5 \% \mathrm{CO}_{2}$. Following infection, the non-internalised bacteria were removed through five rounds of centrifugation at low speed $(1,000 \mathrm{rpm})$ and using HBSS for the resuspension of the B cells after each centrifugation. The cells were then fixed with $4 \%$ paraformaldehyde for $1 \mathrm{~h}$ at room temperature. A cell monolayer was then formed on a glass slide in a Cytospin 3 (Thermo) through the centrifugation of the fixed cells at $700 \mathrm{rpm}$ for $5 \mathrm{~min}$. The monolayer was washed twice with PBS and the cells were permeabilised for 10 min with a $0.1 \%$ Triton X-100 solution in PBS. The cells were then washed twice with PBS and covered with $80 \mathrm{ng}$ of a rhodamine-phalloidin solution (Sigma) per cover slip for $20 \mathrm{~min}$ at room temperature. Excess phalloidin was removed by washing five times with PBS. The labelled preparations were mounted on a glass slide with Vectashield solution (Vector Laboratories) and observed using a confocal laser scanning microscope system attached to a microscope (LSM 510, Zeiss).

\section{Results}

\section{Survival of intracellular bacteria}

To determine whether mycobacteria can replicate in B cells, antibiotic-protection assays were conducted. The S. typhimurium bacteria were completely eliminated by B cells (Figure 1b); in addition, although $M$. smegmatis underwent brief replication during the first $24 \mathrm{~h}$ of infection, an important decrease in the intracellular bacteria was observed starting at $48 \mathrm{~h}$ and through the end of the post-infection kinetics (Figure 1a). S. typhimurium did not present any intracellular replication; in fact, at 6 $\mathrm{h}$ post-infection (Figure 1b), a significant decrease in the bacterial load was observed, which resulted in total bacterial elimination. In contrast, the internalised $M$. tuberculosis exhibited intracellular growth in B cells and sustained exponential growth throughout the experiment (72 $\mathrm{h}$ after infection) (Figure 1a).

\section{Fluid-phase uptake by infected B cells}

Untreated (control) B cells exhibited a very low capability for fluid-phase uptake (Figure 2a-f); however, these cells presented an RFU time- and treatment-dependent increase in fluid-phase uptake under several experimental conditions. The $S$. typhimurium infection induced the highest fluid-phase uptake, with a peak reached after 120 min of infection, but the RFU values were found to decrease thereafter (Figure 2b). M. tuberculosis induced a sustained RFU increase (Figure 2c), but the RFU values were lower than those achieved with S. typhimurium. M. smegmatis triggered the lowest and slowest uptake (Figure 2e). Furthermore, PMA was the best inducer of fluid-phase uptake, but the RFU values were not as high as those reached with S. typhimurium. Similar to the kinetics observed with $S$. typhimurium, after the RFU peak was reached, a decrease in the fluorescence was observed for PMA (Figure 2a). The mycobacterial supernatants induced uptake tendencies that were similar to those 

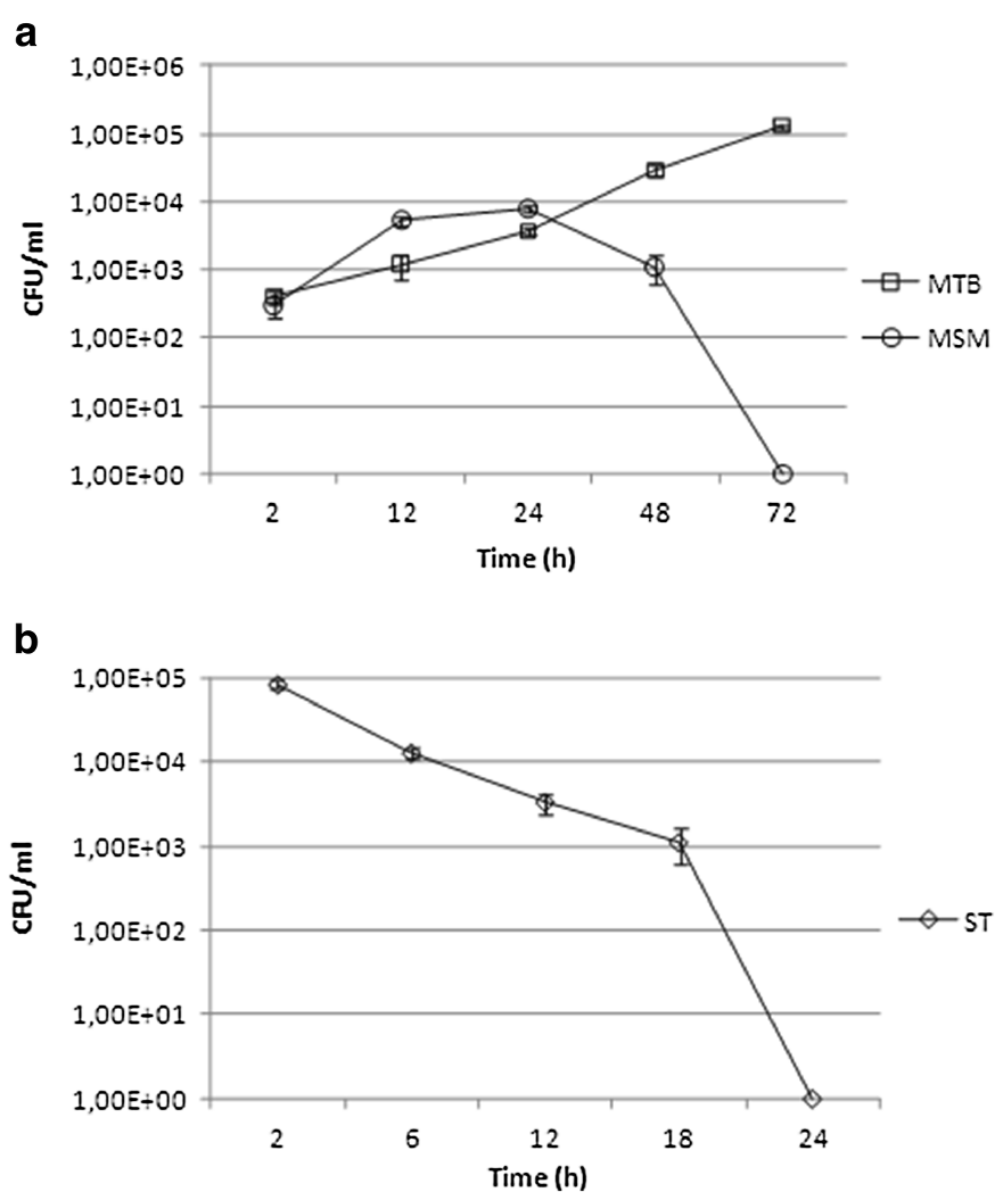

Figure 1 Colony forming units (CFU) of S. typhimurium and mycobacteria in B cells. a) Time-dependent CFU counts of intracellular M. smegmatis (MSM) (circles) and M. tuberculosis (MTB) (squares). The growth of M. smegmatis is controlled by the end of the kinetics, whereas M. tuberculosis survives and multiplies. b) Time-dependent CFU counts of intracellular S. typhimurium (ST). The intracellular growth was rapidly controlled by the B cells compared to the mycobacteria. Each point represents the mean \pm standard error (SE) of triplicate measurements. The experiment presented is representative of three independent repetitions.

observed with their respective bacteria (MTB-SN induced the highest and fastest uptake) (Figures $2 \mathrm{~d}$ and $2 \mathrm{f}$ ). Interestingly, only live bacteria (S. typhimurium, M. tuberculosis, and M. smegmatis) triggered this phenomenon because heat-treated bacteria did not induce any fluidphase uptake (data not shown).

\section{Effect of inhibitors on bacterial and fluid-phase uptake by $B$ cells}

To determine the pathway responsible for the bacterial and fluid-phase uptake that was previously observed in the B cells, several classical endocytic inhibitors were employed [26], including AMIL (macropinocytosis), CD, and WORT (macropinocytosis and phagocytosis). In addition, bacterial infections and soluble treatments (PMA or mycobacterial supernatants) were used in these experiments. The fluid-phase uptake induced during bacterial infections was completely abolished by AMIL, WORT, and CD (Figures 2a through $\mathrm{f}$ ), and this inhibition was observed throughout the experiment. Similarly, the fluid-phase intake triggered by PMA, $M$. tuberculosis, or the $M$. smegmatis supernatant was suppressed by these inhibitors (Figures 2a, 2d and 2f). The inhibition in all these cases was statistically significant. In addition, the bacterial uptake was inhibited with amiloride at all concentrations used (Figure 3). The ST and MSM uptakes were the most affected. Even at the lowest inhibitor concentration used (1 mM), a high uptake inhibition was observed with all bacteria. These observations indicated that macropinocytosis was responsible for the uptake of bacteria into these cells.

\section{Transmission electron microscopy of infected B cells}

To establish the ultrastructural changes that are induced by mycobacteria, the cells were analysed using transmission electron microscopy. The uninfected cells exhibited a round shape, a low cytoplasm/nuclei ratio, and scarce and small membrane projections; therefore, no significant 


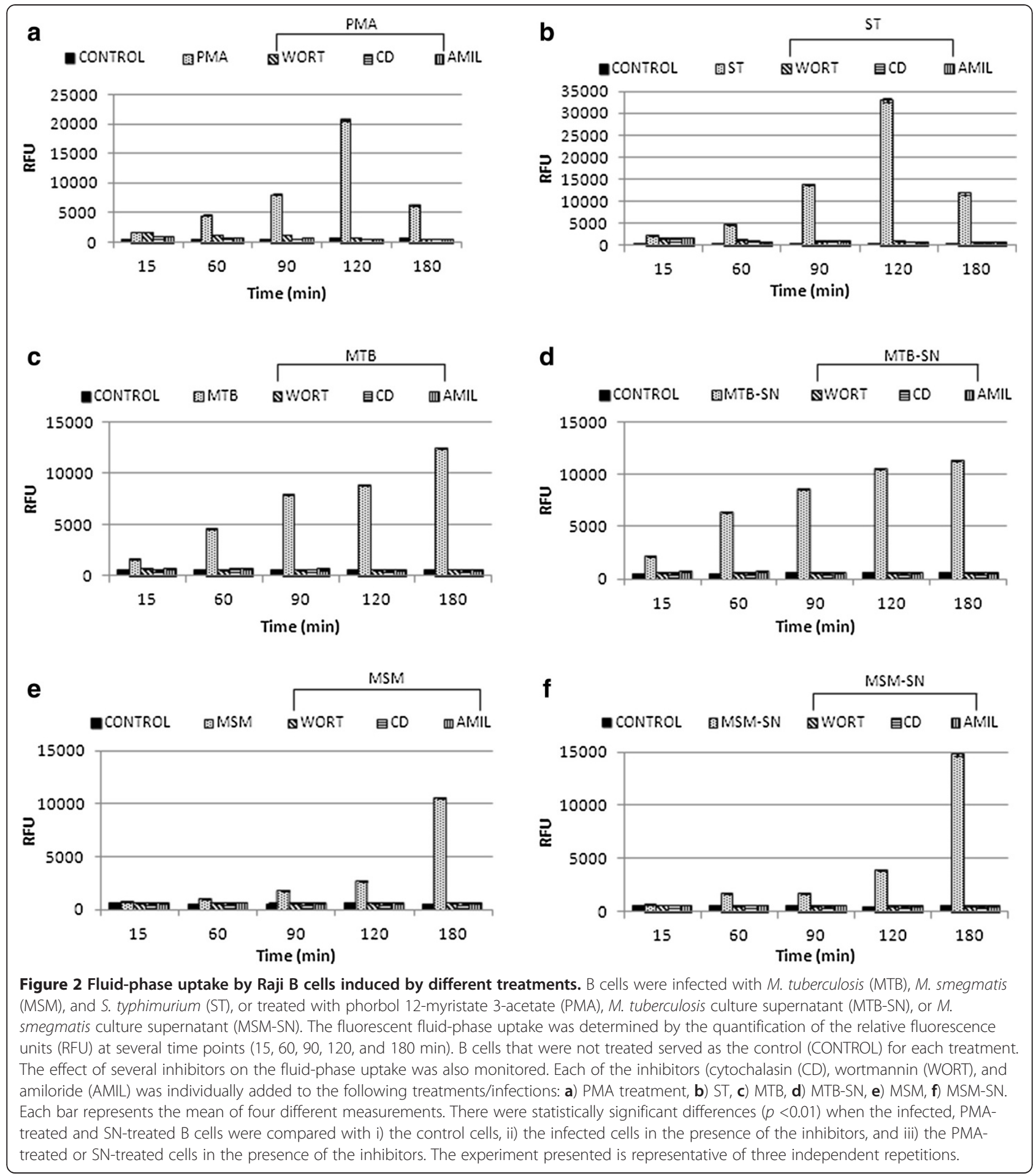

internalisation features were observed (Figures $4 \mathrm{a}$ and $4 \mathrm{~b}$ ). When the cells were infected or treated with soluble components, a number of changes were observed. The PMAtreated cells exhibited a large number of vacuoles or macropinosomes of different sizes (Figures 4c and 4d). As shown in Figure 4e, S. typhimurium induced the formation of membrane extensions, such as lamellipodia. In addition, intracellular bacteria were observed and were found to be surrounded by these membrane projections (Figure 4f). In some Salmonella-infected cells, a number of structures, such as double membrane vacuoles and multilamellar bodies, were observed (Figure 4f). M. smegmatis induced long membrane projections, which surrounded the bacteria (Figure 5a). Some intracellular 


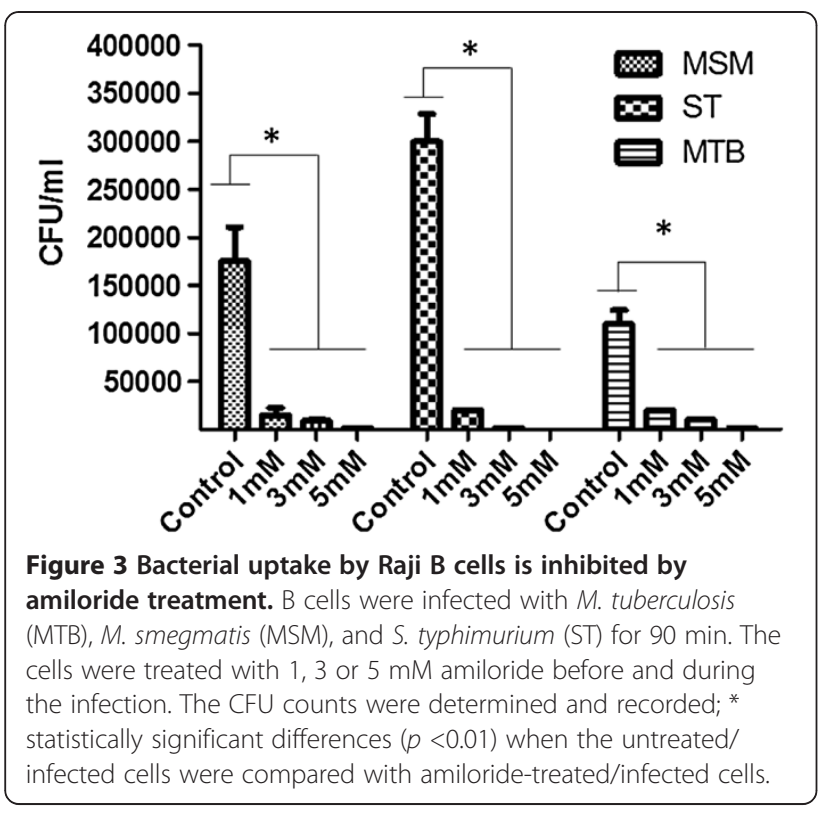

mycobacteria were observed to have cell wall damage (Figure $5 \mathrm{~b}$ ). At $24 \mathrm{~h}$ post-infection, it was difficult to find any internalised bacilli, and the cellular morphology was similar to that of uninfected cells, although some large mitochondria were still observed (Figure 5c). In contrast, major ultrastructural changes due to M. tuberculosis infection were evident: the infected cells contained abundant vacuoles of different sizes and shapes and, in many cases, these vacuoles exhibited an extended and curved shape and were found in close proximity to the nuclei (Figure 5d). In addition, the M. tuberculosis-infected cells showed abundant swollen mitochondria and, frequently, mitochondria that were sequestered into double membrane structures (Figures 5e and 5f). After $24 \mathrm{~h}$ of infection with $M$. tuberculosis, the cells did not recover their basal morphology and still presented abundant vacuoles (Figure 5g). Unlike M. smegmatis and S. typhimurium, intracellular M. tuberculosis replicated well in these cells (Figures $5 \mathrm{~h}$ ) and the bacterial morphology was excellent (5i).

\section{Scanning electron microscopy of infected Raji B cells}

The resting B cells (Figures 6a and $6 \mathrm{~b}$ ) possessed a smooth to slightly irregular membrane. However, drastic changes in the membrane ultrastructure were observed with the different treatments that were administered. PMA, which is known as a classical macropinocytosis inducer, induced the formation of membrane ruffling, filopodia, and lamellipodia that entirely surrounded the cells (Figures $6 \mathrm{c}$ and 6d). M. smegmatis (Figures 6e and 6f) and S. typhimurium (Figures 6i, 6j and 6k) induced a similar phenomenon: membrane ruffling and filopodia formation that completely covered the cell. The bacteria were also found to be attached either to the cell by membrane ruffles (Figures $6 \mathrm{e}$ and $6 \mathrm{j}$ ) or long filopodia (Figures $6 \mathrm{j}$ and $6 \mathrm{i}$ ) or to inside the cell (Figure 6k). In contrast, M. tuberculosis infection mainly induced membrane ruffling (Figures $6 \mathrm{~g}$ and $6 \mathrm{~h}$ ), and the bacilli were trapped by the wide membrane sheets (Figure $6 \mathrm{~g}$ ). All of these images resemble macropinocytic processes, which confirm the TEM observations, the fluid-phase results and the bacterial uptake data that were presented previously.

\section{Cytoskeletal role: actin filaments}

To establish the role of the actin filaments on the mycobacterial internalisation, we performed confocal analyses. The actin filaments were stained with phalloidinrhodamine and the bacteria were labelled with FITC. The uninfected cells presented a peripheral fluorescent label that sustained the spatial cell morphology (Figure 7a). The S. typhimurium-infected cells lost the regular peripheral fluorescent label. After $1 \mathrm{~h}$ of infection, the actin cytoskeletal rearrangements resulting in membrane ruffling were evident on the cell surface, and the attachment of the bacilli to these structures was observed (Figure $7 \mathrm{~b}$ ). After $3 \mathrm{~h}$ of infection, the cells exhibited long actin projections and actin re-distribution (Figure 7c). Additionally, some bacilli were found adhered to the actin organisations that resulted in the lamellipodia formation (Figure 7d). Furthermore, these changes were also observed in cells without any adhered or internalised bacteria (Figure 7b). M. smegmatis infection caused actin rearrangements that could terminate in membrane ruffling, lamellipodia, and filopodia formation. Some cells also showed actin focal spots on the cell surface (Figures $8 \mathrm{a}, 8 \mathrm{~b}$ and $8 \mathrm{c}$ ). After $3 \mathrm{~h}$ of infection, long actin filaments, which are responsible for the formation of membrane filopodia, were present on the cell surface (Figure 8c). M. smegmatis infection-associated actin rearrangements were evident in all of the cells that were present in the preparation, although some cells did not present either adhered or internalised bacilli (Figures 8a, 8b and 8c). M. tuberculosis infection induced actin reorganisation that was responsible for membrane ruffling (Figures $8 \mathrm{~d}, 8 \mathrm{e}$ and $8 \mathrm{f}$ ), although fewer long actin filament formations were observed compared to the other infections (Figures $8 \mathrm{~d}$ and $8 \mathrm{f}$ ). Further, adhered and internalised bacilli were evident after 1 and $3 \mathrm{~h}$ of infection, respectively (Figures $8 \mathrm{~d}$ and 8e). As with all of the other infections, all of the actin cytoskeletal changes were also evident in cells without any adhered or intracellular bacteria (Figure 8f).

\section{Discussion}

The classical B-cell roles include the production of antibodies and cytokines and the generation of immunological memory, which are key factors in the adaptive 

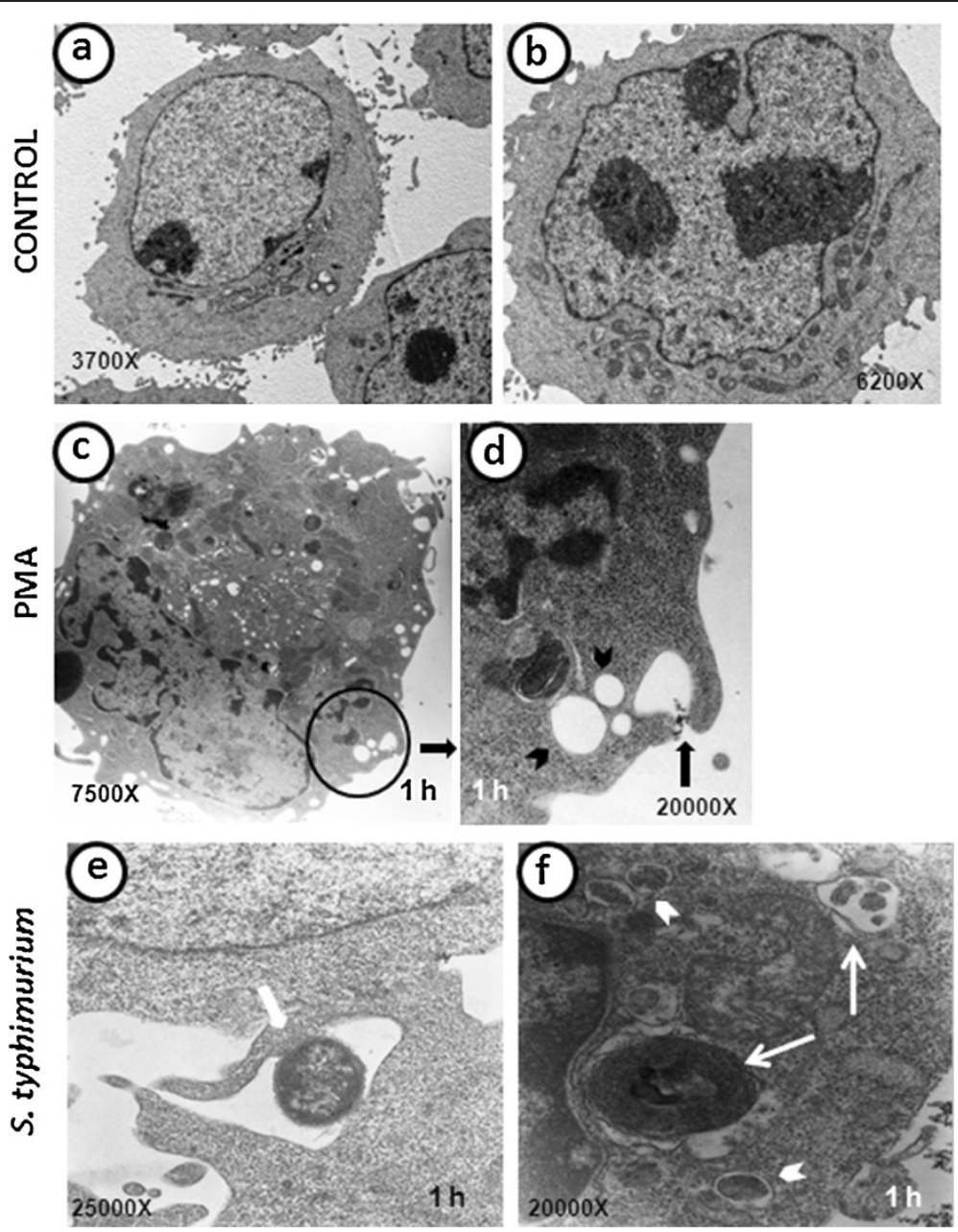

Figure 4 Ultrastructure of B cells infected with S. typhimurium (ST) and stimulated with phorbol 12-myristate 3-acetate (PMA). a-b) Control B cells. c) PMA-stimulated B cell, which has abundant vacuoles of different sizes. d) The field magnification of a PMA-stimulated B cell (circle) shows macropinosome formation (black narrow) and the presence of macropinosomes that are already formed in various sizes (arrowheads). e) Micrograph of S. typhimurium-infected B cell, which shows that the bacillus is surrounded by large membrane extensions (narrow). f) S. typhimurium-infected B cell with internalised bacteria (arrowheads), thin narrows depicts a multilamellar structure (left) and a late degradative autophagic vacuole (LDAV) (right).

immune response. Recently, their role in innate immunity is been increasingly recognised $[27,28]$. The majority of studies that analyse B-cell specific-antigen recognition mainly focus on soluble antigens. B cells have been traditionally considered non-phagocytic cells [29]; therefore, the process of bacterial uptake by $B$ cells has not been extensively documented.

Mature B cells bind specific soluble protein antigens through a unique and restricted BCR [30-32]. At present, it is known that the Antigen-B-cell receptor (Ag-BCR) complex is internalised into clathrin-coated pits, although raft signalling and actin polymerisation are required for efficient receptor mediated-endocytosis [33]. The binding of antigens to the BCR induces cell signalling and triggers changes in the actin cytoskeletal organisation, although these changes are limited to the vicinity of the Ag-bound
BCR and are not generalised throughout the cellular membrane [33,34]. The actin reorganisation after the BCR-antigen engagement is a rapid albeit transient event [34]. The internalised BCR transports the Ag to the endosomal compartments, where it is fragmented and loaded onto nascent $\mathrm{MHC}$ class II proteins for its presentation to T cells [31].

In contrast to BCR-mediated specific soluble-antigen uptake, we studied the features of non-specific bacterial uptake by B cells. In our study, B lymphoblast cells of the Raji cell line (Burkitt's lymphoma) were infected with two Mycobacterium species and with S. typhimurium, and the resultant cellular membrane changes and cytoskeletal reorganisation events were analysed. We previously reported that macropinocytosis is the mechanism responsible for the internalisation of mycobacteria into the lung 

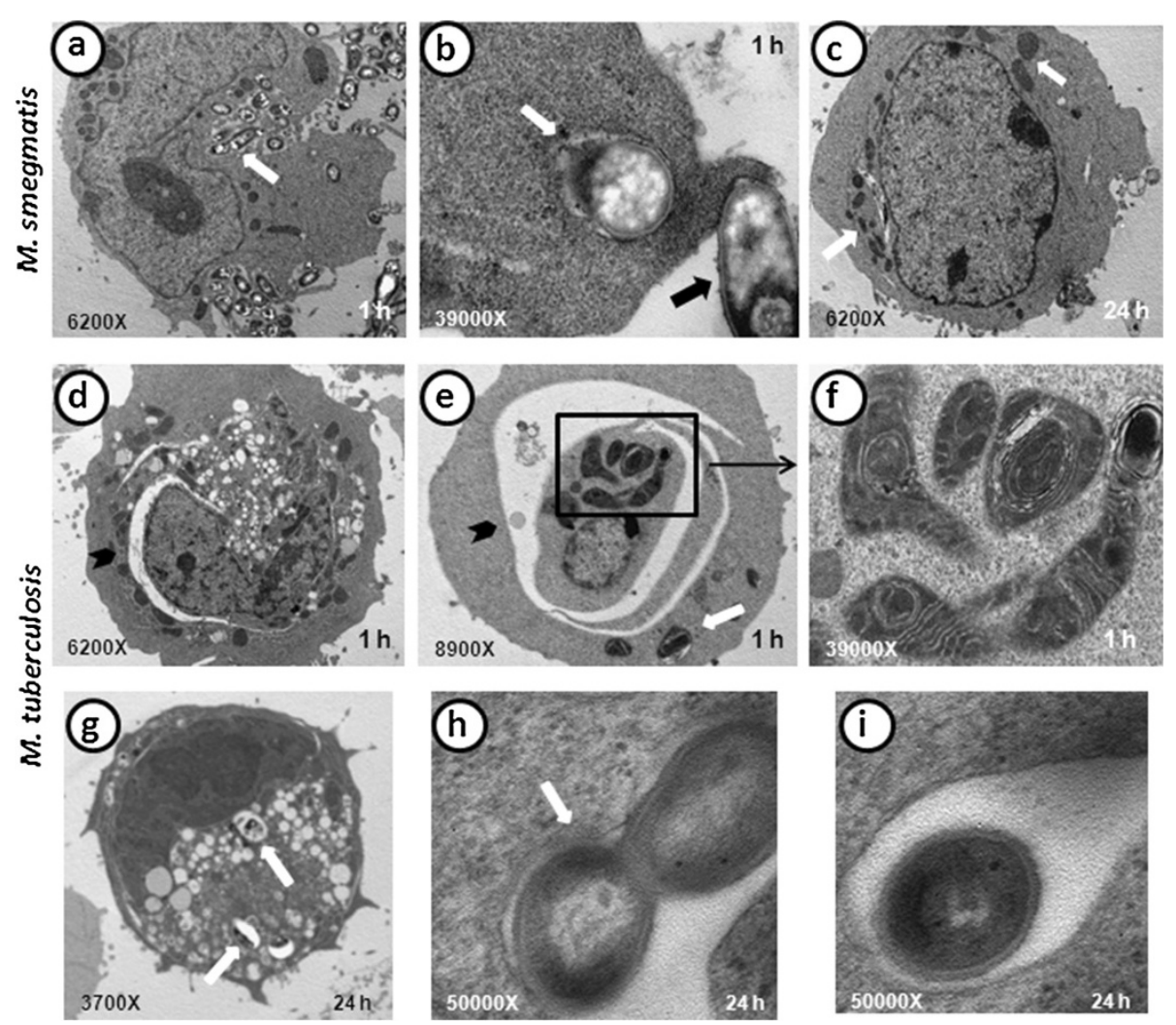

Figure 5 Ultrastructure of B cells infected with $\boldsymbol{M}$. smegmatis (MSM) and $\boldsymbol{M}$. tuberculosis (MTB). a) MSM-infected B cell with abundant internalised bacilli (white arrow) after $1 \mathrm{~h}$ of infection. b) MSM-infected B cell after $1 \mathrm{~h}$ of infection, which shows the binding of a bacillus to a lamellipodium (black arrow) and the destruction of an intracellular bacillus contained in a vacuole (white arrow). c) MSM-infected B cell at $24 \mathrm{~h}$ post-infection, which shows that the cell morphology was recovered and that no internalised bacilli were present, although some swollen mitochondria were still observed (white arrows). d-e) After $1 \mathrm{~h}$ of infection, a B cell infected with MTB exhibits a large number of alterations, abundant vacuoles, swollen mitochondria, internalised mycobacteria (white arrow), and "curved vacuoles" (black arrowheads). f) Magnification of a B cell infected with MTB (square), which shows that some of the altered mitochondria are in the process of forming double to multi-membrane vacuoles (autophagy-like vacuoles). g) B cell infected with MTB for $24 \mathrm{~h}$ shows intracellular bacilli in vacuoles (white arrows), abundant vacuoles, and an electro-dense cellular nucleus, which suggests strong damage. h) Replicating mycobacteria in spacious vacuole (white arrow) formed in a B cell infected with MTB for $24 \mathrm{~h}$. g) Detail of MTB bacillus in a spacious vacuole after $24 \mathrm{~h}$ of B cell infection.

epithelial A549 cell line [18,19] and into endothelial cells [35]. Therefore, considering that B lymphocytes have been recognised as classical non-phagocytic cells [29], we sought to establish whether mycobacteria were able to induce macropinocytic internalisation in B cells. In our design, the infections were conducted with B cells in suspension; to avoid the spreading feature that is commonly observed in these cells, we did not plate Raji cells on any cell surface that was either uncovered or covered with any extracellular matrix ligands or antibodies [36,37].

Our observations revealed that the B cells were readily infected by the three bacteria that were studied and that the infections induced relevant changes in the cellular membrane during bacterial internalisation (Figure 6). $M$. smegmatis is considered a non-pathogenic mycobacteria; however, it was able to induce important membrane changes that were characterised by abundant filopodia and lamellipodia formation (Figure 6e and $6 \mathrm{f}$ ) and were similar to those triggered by PMA (Figures $6 \mathrm{c}$ and $6 \mathrm{~d}$ ). B cells that were treated with the supernatant from the bacterial cultures (mycobacteria were removed by centrifugation and filtration) exhibited the same ultrastructural changes (data not shown). M. smegmatis was readily internalised; in fact, some cells internalised a large number of the mycobacteria (Figure 5a). M. smegmatis exhibited a transient multiplication, which was revealed by the counting of CFU 12 and $24 \mathrm{~h}$ postinfection (Figure 1a). However, by 48 and $72 \mathrm{~h}$, the mycobacteria were eliminated. After $24 \mathrm{~h}$ of infection, no evident intracellular mycobacteria were observed on the TEM images, and the B cell morphology was similar to that of uninfected cells (Figure 5c). Intravacuolar mycobacteria destruction was clearly observed, and partial destruction of the bacterial cell wall was evident (Figure 5b). The results from the analysis of mycobacterial intracellular elimination, membrane protrusion 


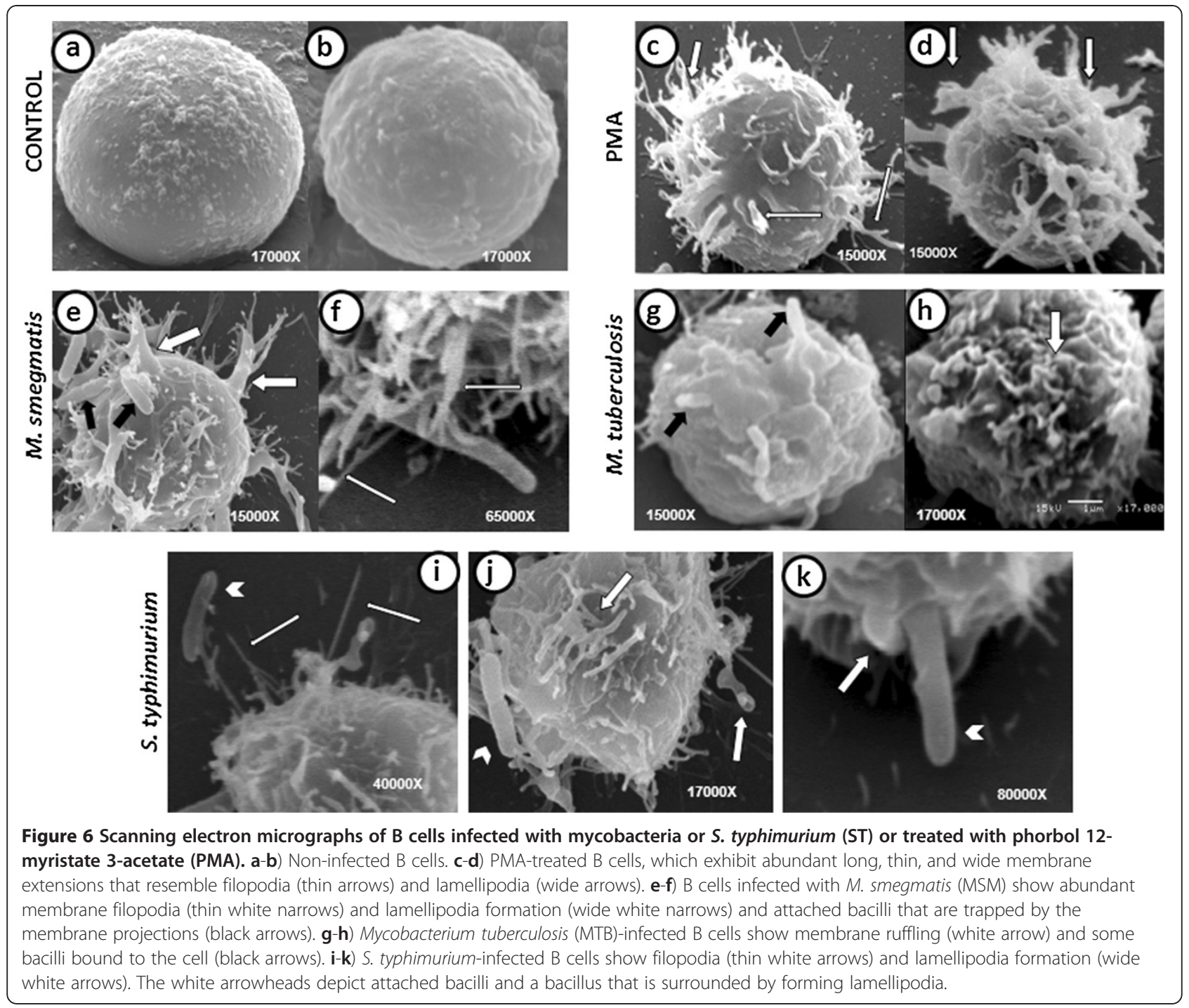

formation, and cytoskeleton rearrangements during bacterial uptake resemble those observed in the infection of epithelial and endothelial cells by $M$. smegmatis $[19,35]$, although $M$. smegmatis induced significantly fewer changes in endothelial cells. To our knowledge, there are no other reports of B cell infection by M. smegmatis; therefore, this study is the first description of this subject.

The M. tuberculosis infection of B cells showed some differences with the effect of M. smegmatis and S. typhimurium infections. M. tuberculosis has previously demonstrated the capability to invade several cell types, including epithelial [18,38], fibroblast [39], and endothelial cells $[35,40]$. The cellular membrane protrusions formed during M. tuberculosis internalisation have been described in some of these cells $[18,35,40]$. In B cells, membrane protrusions were also observed during $M$. tuberculosis uptake. However, these protrusions were different from those observed with M. smegmatis and S. typhimurium infections or with PMA treatment; these appeared to be wider and shorter compared with those formed during the other treatments/infections (Figures $6 \mathrm{~g}$ and $6 \mathrm{~h}$ ). In addition, no evident filopodia formation was observed during $M$. tuberculosis infection, and the protrusions were more similar to ruffles. The actin cytoskeleton sustained these membrane protrusions (Figures 8e and 8f), although the actin filaments were shorter compared to those formed during PMA treatment and M. smegmatis or S. typhimurium infection. Of the three bacteria utilised for the infection of B cells, only $M$. tuberculosis was able to survive and multiply intracellularly (Figure 1). In an earlier study of $M$. tuberculosis uptake by human-transformed B cells [14], the authors described the formation of membrane protrusions during mycobacterial infection that were similar to those described by our group. The authors also demonstrated the presence of mycobacteria in spacious vacuoles and the 

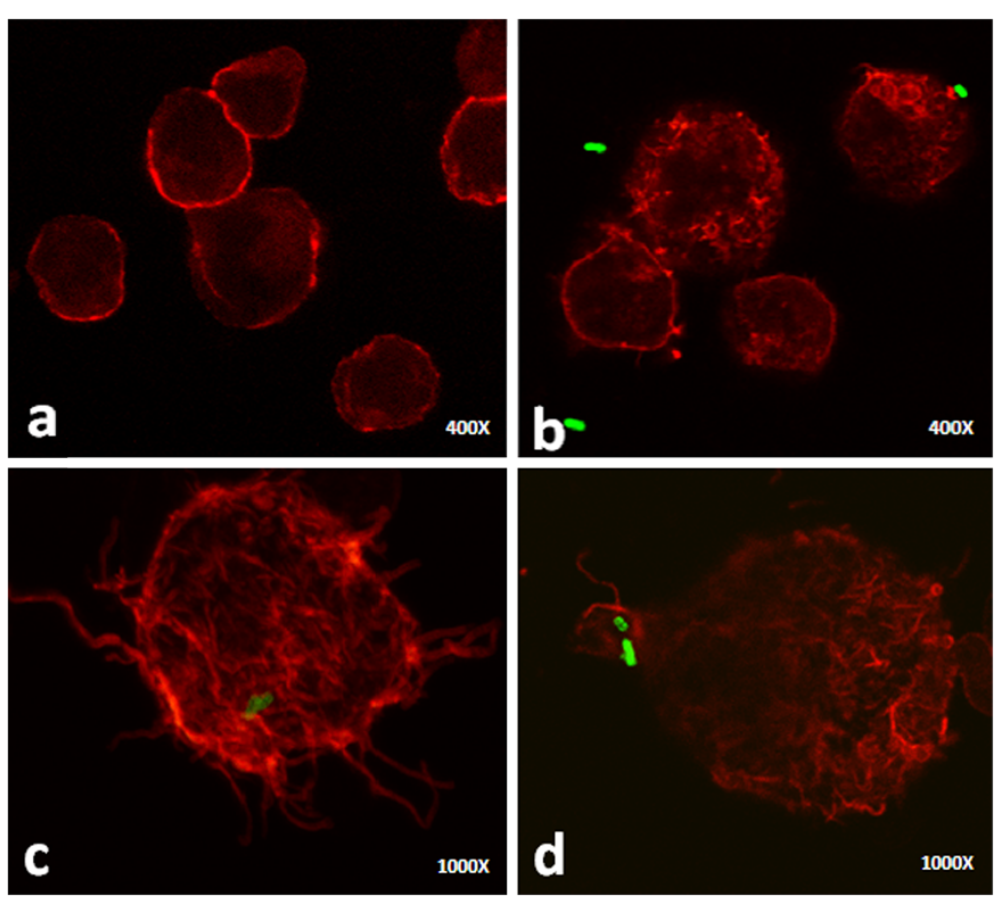

Figure 7 Confocal images of uninfected and S. typhimurium (ST)-infected B cells. The actin filaments were labelled with rhodaminephalloidin and the bacteria were stained with fluorescein isothiocyanate (FITC). a) Uninfected cells present peripheral and homogeneous fluorescent staining. b) One $\mathrm{h}$ after infection, S. typhimurium induced actin cytoskeletal rearrangements that are responsible for membrane ruffling; in addition, a bacillus that is attached to these structures was observed (upper right corner). c-d) After $3 \mathrm{~h}$ of infection, longer actin projections and actin redistribution were observed, and some bacilli were found inside the B cell (c) or surrounded by actin organisations (d).
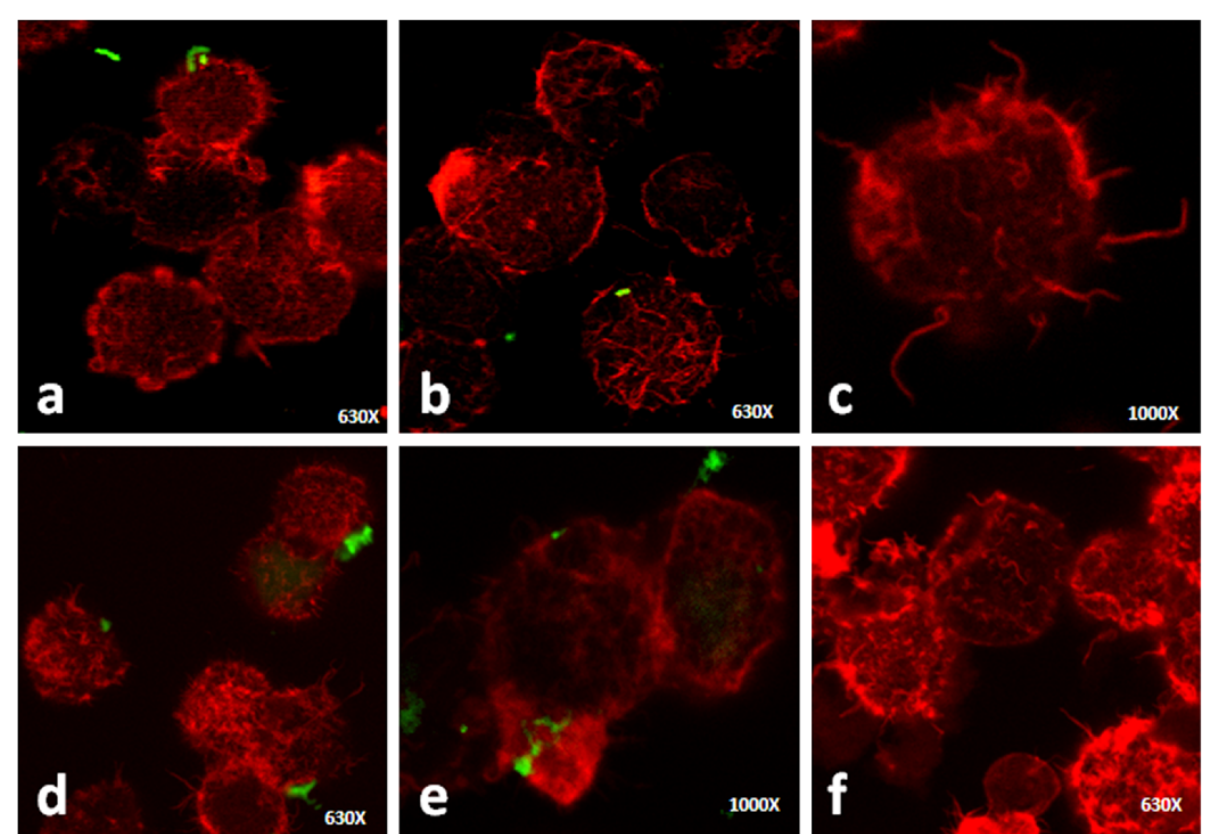

Figure 8 Confocal images of B cells infected with mycobacteria. The actin filaments were labelled with rhodamine-phalloidin and the bacteria were stained with fluorescein isothiocyanate (FITC). a) M. smegmatis (MSM) infection caused evident actin rearrangements within $1 \mathrm{~h}$ of infection; a mycobacterium was observed attached to the cell. b-c) After $3 \mathrm{~h}$ of infection with MSM, intracellular bacteria were observed (b) and long actin filaments were evident (c). d) M. tuberculosis (MTB) infection induced actin reorganisation after $1 \mathrm{~h}$ of infection, and bacilli attached to the cells were observed; e-f) B cells, after $3 \mathrm{~h}$ of infection with MTB, presented actin cytoskeletal changes in cells without any adhered or intracellular bacteria. 
presence of abundant mitochondria in infected cells. The authors indicated that the internalisation of live $M$. tuberculosis by B cells results in the presentation of the mycobacterial antigen to $\mathrm{T}$ cells. A number of characteristic structures were observed in B cells that were infected with M. tuberculosis, including "curved vacuoles" with arched or crescent shapes (Figures $5 \mathrm{~d}$ and $5 \mathrm{e}$ ), which contain amorphous material. Because these structures were not observed with the other infections, they appear to be characteristic of $M$. tuberculosis infection.

In our study, we were unable to observe Salmonellainduced filaments (SIFs), which are the hallmark organelles in which the bacteria multiply in epithelial cells $[41,42]$. This observation might be the result of the rapid elimination of Salmonella from the B cells. To our knowledge, there is currently no description of SIF formation in Salmonella-infected B cells.

B-cell infection by $S$. typhimurium has been previously reported $[29,43,44]$. It is known that S. typhimurium is internalised through macropinocytosis in several cell models, such as epithelial cells and macrophages [45,46]. It was recently demonstrated that $S$. typhimurium can infect B cells by macropinocytosis [20]. Thus, we utilised the Salmonella infection of B cells as a positive control to corroborate that the process induced during mycobacterium internalisation by B cells was macropinocytosis. All of the features observed during B cell infection by Salmonella were consistent with the phenomenon of macropinocytosis, including the membrane protrusion formation (Figure 6j), actin involvement (Figures $7 \mathrm{~b}, 7 \mathrm{c}$ and $7 \mathrm{~d}$ ), and spacious vacuole formation (Figure $4 \mathrm{e}$ and 4f) [46-48]. Therefore, due the morphological evidence and the inhibition of bacterial internalisation by amiloride, we can conclude that S. typhimurium induced macropinocytosis for its internalisation into the Raji B cell, which confirms the recent findings on the internalisation of S. typhimurium into mouse primary B cells [20]. During macropinocytosis, large volumes of fluid-phase are found in spacious vacuoles known as macropinosomes; therefore, the quantification of the fluorescent-labelled fluid-phase could be an indirect measurement of the macropinocytosis that is triggered by some inducer $[49,50]$. In this context, S. typhimurium induced the highest uptake by B cells. The level of internalisation of S. typhimurium was higher than that achieved with PMA, which is considered an efficient inducer of macropinocytosis [25]. Both of the mycobacteria induced a lower uptake; however, in contrast to Salmonella or PMA, we did not observe any reduction in the fluorescence uptake throughout the experiment. The use of pharmacological inhibitors complements the study of endocytosis and aids in the elucidation of the endocytic processes that occur in different cells $[26,49,50]$. In this study, we found that, during Salmonella or mycobacteria infections, the fluid-phase uptake was abolished by CD,
WORT, and AMIL, confirms the involvement of the cytoskeleton during the infection, the participation of PI-3K, and the phenomenon of macropinocytosis as the process that is responsible for the bacterial internalisation. Interestingly, the M. tuberculosis and M. smegmatis culture supernatants (obtained during the log-phase growth of the bacteria) were able to induce the same level of fluid-phase uptake as the live bacteria. Furthermore, the supernatant fluid-phase uptake was inhibited by all of the inhibitors, which suggests that the soluble factors that are produced by these bacteria are able to induce macropinocytosis and is consistent with previous studies that have suggested this phenomenon in other cell types $[18,19]$.

Different from other B-cell models [29,43,44], S. typhimurium was eliminated by the Raji B cells (Figure 1b), no replicating intracellular bacteria were observed in the Salmonella-containing vacuoles of these B cells, and no SIF structures were induced in the cells during the Salmonella productive infection [41,42]. Instead, we observed (Figure 4f) non-replicating bacteria, some of which were in the process of being destroyed, multilamellar bodies, and some late degradative autophagic vacuoles (LDAV) [51]; the presence of these structures suggests that autophagy was in progress, which could be partly responsible for the containment of the Salmonella growth [52], although this observation should be analysed in more detail. In contrast to the Raji B-cell line, the Ramos B-cell line can internalise only Salmonella that is bound to the specific anti-Salmonella antibody; thus, the BCR-mediated internalisation in these cells allowed Ag presentation, IgM anti-Salmonella production, and Salmonella intracellular survival [29].

B cells from early vertebrates, such as teleost fish, are able to internalise bacteria and exert microbicidal abilities [10]. In this study, Raji B cells, like the B cells from early vertebrates, were able to control S. typhimurium and M. smegmatis but not M. tuberculosis. The precise antimicrobial mechanisms that are exerted by B cells from cell lines or primary cells are not yet well known. To date, among the possible antimicrobial mechanisms, nitric oxide (NO) is believed to be responsible for the control of pathogen growth by B cells. The B1 subset of B lymphocytes constitutively expresses the mRNA of inducible nitric oxide synthase (iNOS) and produces $\mathrm{NO}$ prior to and during Cryptococcus neoformans infection, which contributes to the elimination of the pathogen [53]. The B1 cells also produce NO under TLR stimulation, which suggests that these cells have a role in nonspecific, cell-mediated immunity against pathogens [54]. Novel recent evidence suggests that $B$ cells may also produce defensins in response to TLR stimulation. For example, the stimulation of $B$ cells with CpG-DNA induces the production of $\beta$-defensin 2 [55]. The scarcity of evidence on the $\mathrm{B}$ cell mechanisms that are involved 
in the destruction of pathogens and on the precise role of $\mathrm{B}$ cells in the innate and specific response against mycobacterial infection makes this an interesting field of research.

\section{Conclusions}

In this manuscript, we describe the events that occurred during the internalisation of three different bacteria into a B lymphoblast cell line (Raji cell line). M. smegmatis, M. tuberculosis and S. typhimurium were readily internalised by Raji B cells as early as $1 \mathrm{~h}$ post-infection, and their uptake was inhibited in the presence of amiloride. During mycobacteria and Salmonella uptake, the B cells formed lamellipodia, ruffling and filopodia. After uptake, many spacious vacuoles or macropinosomes of different sizes were observed. The fluid-phase uptake that occurs during Salmonella or mycobacteria internalisation was abolished by amiloride, cytochalasin D or wortmannin, which confirms the involvement of the cytoskeleton during the internalisation, the participation of PI-3K, and the triggering of macropinocytosis during bacterial uptake. Death mycobacteria did not induce fluid-phase uptake in B cells. The secreted products in a $M$. tuberculosis and M. smegmatis culture were able to induce the same level of fluid-phase uptake as the live bacteria, and the supernatant-induced fluid-phase uptake was inhibited by all of the inhibitors, which indicates that the soluble factors that are produced by these bacteria are able to induce macropinocytosis. The B cell cytoskeleton underwent crucial rearrangements during bacterial internalisation, which signifies that the cytoskeleton plays a role during macropinocytosis. M. smegmatis and S. typhimurium were eliminated by the Raji B cells; however, $M$. tuberculosis was able to survive and multiply in these cells, which suggests that the induction of macropinocytosis does not warrant bacterial elimination or survival.

\section{Abbreviations \\ (MTB): Mycobacterium tuberculosis; (MSM): Mycobacterium smegmatis; (ST): Salmonella typhimurium; (AMIL): Amiloride; (WORT): Wortmannin; (CD): Cytochalasin D; (SN): Culture supernatant; (SIFs): Salmonella-induced filaments; (RFU): Relative fluorescent units; (CFUs): Colony forming units.}

\section{Competing interests}

The authors of this study have no conflicts of interest to report.

\section{Authors' contributions}

$B E G P, J J D C L$, JICS, ARMD and ACL carried out the experiments and prepared the samples for electron microscopy observation. ADHP and HVC processed and analysed the TEM samples. EGL participated in the design of the study and contributed to the draft and review of the manuscript. BEGP helped draft the manuscript and edited the figures. JLH conceived the study, participated in its design and coordination and coordinated the drafting of the manuscript. All authors read and approved the final manuscript.

\section{Acknowledgements}

This work was supported by CONACYT (project SEP-2004-C01) and SIP/IPN (projects 20121279 and 20121160). BEGP, JLH and EGL received fellowships from COFAA and EDI. JLH and EGL are SNI fellows. JJCL, ARMD, ACL and JICS would like to acknowledge CONACYT and PIFI for their fellowships. JICS is also an ICYT-DF fellow. The authors would also like to acknowledge the Electron Microscopy Central of ENCB/IPN for technical assistance.

\section{Author details}

${ }^{1}$ Immunology Department, Escuela Nacional de Ciencias Biológicas, Instituto Politécnico Nacional, Prolongación de Carpio y Plan de Ayala s/n, Z.P. 11340, México, D.F, México. ${ }^{2}$ Electron Microscopy Laboratory, Instituto Nacional de Rehabilitación, Av. México-Xochimilco No. 289, Col. El Arenal de Guadalupe, Tlalpan, México, D.F, México.

Received: 27 January 2012 Accepted: 12 October 2012

Published: 31 October 2012

\section{References}

1. Lund FE, Garvy BA, Randall TD, Harris DP: Regulatory roles for cytokineproducing $B$ cells in infection and autoimmune disease. Curr Dir Autoimmun 2005, 8:25-54.

2. Batista FD, Iber $D$, Neuberger MS: $B$ cells acquire antigen from target cells after synapse formation. Nature 2001, 411:489-494.

3. Gupta N, DeFranco AL: Lipid rafts and B cell signaling. Semin Cell Dev Biol 2007, 18:616-626.

4. Putnam MA, Moquin AE, Merrihew M, Outcalt C, Sorge E, Caballero A, Gondré-Lewis TA, Drake JR: Lipid raft-independent B cell receptormediated antigen internalization and intracellular trafficking. J Immunol 2003, 170:905-912.

5. Chiron D, Bekeredjian-Ding I, Pellat-Deceunynck C, Bataille R, Jego G: Tolllike receptors: lessons to learn from normal and malignant human $B$ cells. Blood 2008, 112:2205-2213.

6. Kato M, McDonald KJ, Khan S, Ross IL, Vuckovic S, Chen K, Munster D, MacDonald KP, Hart DN: Expression of human DEC-205 (CD205) multilectin receptor on leukocytes. Int Immunol 2006, 18:857-869.

7. Won WJ, Bachmann MF, Kearney JF: CD36 is differentially expressed on B cell subsets during development and in responses to antigen. J Immunol 2008, 180:230-237.

8. Rappocciolo G, Piazza P, Fuller CL, Reinhart TA, Watkins SC, Rowe DT, Jais M, Gupta P, Rinaldo CR: DC-SIGN on lymphocytes is required for transmission of HIV-1 to T lymphocytes. PLoS Pathog 2006, 2:0691-0704.

9. Rappocciolo G, Hensler HR, Jais M, Reinhart TA, Pegu A, Jenkins FJ, Rinaldo CR: Human herpesvirus 8 infects and replicates in primary cultures of activated B lymphocytes through DC-SIGN. J Virol 2008, 82:4793-4806.

10. Li J, Barreda DR, Zhang YA, Boshra H, Gelman AE, Lapatra S, Tort L, Sunver JO: B lymphocytes from early vertebrates have potent phagocytic and microbicidal abilities. Nat Immunol 2006, 7:1116-1124.

11. Krocova Z, Hârtlova A, Souckova D, Zivna L, Kroca M, Rudolf E, Macela A, Stulik J: Interaction of B cells with intracellular pathogen Francisella tularensis. Microb Pathog 2008, 45:79-85.

12. Vidard L, Kovacsovics-Bankowski M, Kraeft SK, Chen LB, Benacerraf B, Rock $\mathrm{KL}$ : Analysis of MHC class II presentation of particulate antigens of lymphocytes B. J Immunol 1996, 156:2809-2818.

13. Barral P, Eckl-Dorna J, Harwood NE, De Santo C, Salio M, Illarionov P, Besra GS, Cerundolo V, Batista FD: B cell receptor-mediated uptake of CD1drestricted antigen augments antibody responses by recruiting invariant NKT cell help in vivo. Proc Natl Acad Sci USA 2008, 105:8345-8350.

14. Lombardi G, del Gallo F, Vismara D, Piccolella E, De Martino C, Garzelli C, Puglisi C, Colizzi V: Epstein-Barr virus-transformed B cells process and present Mycobacterium tuberculosis particulate antigens to T-cell clones. Cell Immunol 1987, 107:281-292.

15. Verjans GM, Ringrose JH, van Alphen L, Feltkamp TE, Kusters JG: Entrance and survival of Salmonella typhimurium and Yersinia enterocolitica with human B- and T-cell lines. Infect Immun 1994, 62:2229-2235.

16. Shibuya A, Sakamoto N, Shimizu Y, Shibuya K, Osawa M, Hiroyama T, Eyre HJ, Sutherland GR, Endo Y, Fujita T, Miyabayashi T, Sakano S, Tsuji T, Nakayama E, Phillips JH, Lanier LL, Nakauchi H: Fc alpha/mu receptor mediates endocytosis of IgM-coated microbes. Nat Immunol 2000, 1:441-446.

17. Menon A, Shroyer ML, Wampler JL, Chawan CB, Bhunia AK: In vitro study of Listeria monocytogenes infection to murine primary and human transformed B cells. Comp Immunol Microbiol Infect Dis 2003, 26:157-174. 
18. Garcia-Perez BE, Mondragon-Flores R, Luna-Herrera J: Internalization of Mycobacterium tuberculosis by macropinocitosis in non-phagocytic cells. Microb Pathog 2003, 35:49-55.

19. Garcia-Perez BE, Hernandez-Gonzalez JC, Garcia-Nieto S, Luna-Herrera J: Internalization of a non-pathogenic micobacteria by macropinocitosis in human alveolar epitelial A549 cells. Microb Pathog 2008, 45:1-6.

20. Rosales-Reyes R, Pérez-López A, Sánchez-Gómez C, Hernández-Mote RR, Castro-Eguiluz D, Ortiz-Navarrete V, Alpuche-Aranda CM: Salmonella infects $B$ cells by macropinocytosis and formation of spacious phagosomes but does not induce pyroptosis in favor of its survival. Microb Pathog 2012, 52:367-74.

21. West MA, Bretscher MS, Watts C: Distinct endocytotic pathways in epidermal growth factor-stimulated human carcinoma A431 cells. J Cell Biol 1989, 109:2731-9.

22. Koivusalo M, Welch C, Hayashi H, Scott CC, Kim M, Alexander T, Touret N, Hahn KM, Grinstein S: Amiloride inhibits macropinocytosis by lowering submembranous $\mathrm{pH}$ and preventing Rac1 and Cdc42 signaling. J Cell Biol 2010, 188:547-63.

23. Brenner SL, Korn ED: The effects of cytochalasins on actin polymerization and actin ATPase provide insights into the mechanism of polymerization. J Biol Chem 1980, 255:841-4.

24. Araki N, Johnson MT, Swanson JA: A role for phosphoinositide 3-kinase in the completion of macropinocytosis and phagocytosis by macrophages. J Cell Biol 1996, 135:1249-60.

25. Swanson JA: Phorbol esters stimulated macropinocytosis and solute flow through macrophages. J Cell Sci 1989, 94:135-142.

26. Ivanov Al: Pharmacological inhibition of endocytic pathways: is it specific enough to be useful? Meth Mol Biol 2008, 440:15-33.

27. Lopez JD, Mariano M: B-1 cell: the precursor of a novel mononuclear phagocyte with immuno-regulatory properties. An Acad Bras Cienc 2009, 81:489-496.

28. Russo RT, Mariano M: B-1 cell protective role in murine primary Mycobacterium bovis bacillus Calmette-Guerin infection. Immunobiology 2010, 215:1005-1014.

29. Souwer Y, Griekspoor A, Jorritsma T, de Wit J, Janssen H, Neefjes J, van Ham SM: B cell receptor-mediated internalization of salmonella: a novel pathway for autonomous B cell activation and antibody production. J Immunol 2009, 182:7473-7481.

30. Vidard L, Kovacsovics-Bankowski M, Kraeft SK, Chen LB, Benacerraf B, Rock $\mathrm{KL}$ : Analysis of MHC class II presentation of particulate antigens of lymphocytes B. J Immunol 1996, 156:2809-2818.

31. Malhotra S, Kovats S, Zhang W, Coggeshall KM: B cell antigen receptor endocytosis and antigen presentation to $T$ cells require Vav and dynamin. J Biol Chem 2009, 284:24088-24097.

32. Jang $C$, Machtaler $S$, Matsuuchi $L$ : The role of $\mathrm{lg}-\mathrm{a} / \mathrm{\beta}$ in $\mathrm{B}$ cell antigen receptor internalization. Immunol Lett 2010, 134:75-82.

33. Stoddart A, Jackson AP, Brodsky FM: Plasticity of B cell receptor internalization upon conditional depletion of clathrin. Mol Biol Cell 2005, 16:2339-2348.

34. Sharma S, Orlowski G, Song W: Btk regulates B cell receptor-mediated antigen processing and presentation by controlling actin cytoskeleton dynamics in B cells. J Immunol 2009, 182:329-339.

35. García-Pérez BE, Villagómez-Palatto DA, Castañeda-Sánchez Jl, Coral-Vázquez RM, Ramírez-Sánchez I, Ordoñez-Razo RM, Luna-Herrera J: Innate response of human endothelial cells infected with mycobacteria. Immunobiology 2011, 216:925-935.

36. McQuade KJ, Rapraeger AC: Syndecan-1 transmembrane and extracellular domains have unique and distinct roles in cell spreading. J Biol Chem 2003, 278:46607-46615.

37. Tse KW, Dang-Lawson M, Lee RL, Vong D, Bulic A, Buckbinder L, Gold MR: B cell receptor-induced phosphorylation of Pyk2 and focal adhesion kinase involves integrins and the Rap GTPases and is required for $\mathrm{B}$ cell spreading. J Biol Chem 2009, 284:22865-22877.

38. Bermudez LE, Shelton K, Young LS: Comparison of the ability of Mycobacterium avium, M. smegmatis and M. tuberculosis to invade and replicate within HEp-2 epithelial cells. Tuber Lung Dis 1995, 76:240-247.

39. Rastogi N, Labrousse V, de Sousa JP: Mycobacterial growth and ultrastructure in mouse L-929 fibroblasts and bone marrow-derived macrophages: evidence that infected fibroblasts secrete mediators capable of modulating bacterial growth in macrophages. Curr Microbiol 1992, 25:203-213.
40. Jain SK, Paul-Satyaseela M, Lamichhane G, Kim KS, Bishai WR: Mycobacterium tuberculosis invasion and traversal across an in vitro human blood-brain barrier as a pathogenic mechanism for central nervous system tuberculosis. J Infect Dis 2006, 193:1287-1295.

41. Garcia-del Portillo F, Zwick MB, Leung KY, Finlay BB: Salmonella induces the formation of filamentous structures containing lysosomal membrane glycoproteins in epithelial cells. Proc Natl Acad Sci USA 1993, 90:10544-10548.

42. Kerr MC, Wang JT, Castro NA, Hamilton NA, Town L, Brown DL, Meunie FA, Brown NF, Stow JL, Teasdale RD: Inhibition of the Ptdlns(5) kinase PIKfyve disrupts intracellular replication of Salmonella. EMBO J 2010, 29:1331-1347.

43. Geddes K, Cruz F, Heffron F: Analysis of cells targeted by Salmonella type III secretion in vivo. Plos Pathog 2007, 3(12):e196.

44. Castro-Eguiluz D, Pelayo R, Rosales-Garcia V, Rosales-Reyes R, AlpucheAranda C, Ortiz-Navarrete V: B cell precursors are targets for Salmonella infection. Microb Pathog 2009, 47:52-56.

45. Mills SD, Finlay BB: Comparison of Salmonella typhi and Salmonella typhimurium invasion, intracellular growth and localization in cultured human epithelial cells. Microb Pathog 1994, 17:409-423.

46. Alpuche-Aranda CM, Racoosin EL, Swanson JA, Miller SI: Salmonella stimulate macrophage macropinocytosis and persist within spacious phagosomes. J Exp Med 1994, 179:601-608.

47. Garcia-del Portillo F, Finlay BB: Salmonella invasion of nonphagocytic cells induces formation of macropinosomes in the host cell. Infect Immun 1994, 62:4641-4645.

48. Kerr MC, Teasdale RD: Defining macropinocytosis. Traffic 2009, 10:364-371.

49. Araki N, Hamasaki M, Egami Y, Hatae T: Effect of 3-methyladenine on the fusion process of macropinosomes in EGF-stimulated A431 cells. Cell Struct Funct 2006, 31:145-57.

50. Hacker U, Albrecht R, Maniak M: Fluid-phase uptake by macropinocytosis in Dictyostelium. J Cell Sci 1997, 110:105-112.

51. Eskelinen EL: Maturation of autophagic vacuoles in Mammalian cells. Autophagy 2005, 1:1-10.

52. Jia K, Thomas C, Akbar M, Sun Q, Adams-Huet B, Gilpin C, Levine B: Autophagy genes protect against Salmonella typhimurium infection and mediate insulin signaling-regulated pathogen resistance. Proc Natl Acad Sci USA 2009, 106:14564-14569.

53. Ghosn EE, Russo M, Almeida SR: Nitric oxide-dependent killing of Cryptococcus neoformans by B-1-derived mononuclear phagocyte. J Leukoc Biol 2006, 80:36-44.

54. Tumurkhuu G, Koide N, Dagvadorj J, Noman AS, Khuda II, Naiki Y, Komatsu T, Yoshida T, Yokochi T: B1 cells produce nitric oxide in response to a series of toll-like receptor ligands. Cell Immunol 2010, 261:122-127.

55. Han SH, Kim YE, Park JA, Park JB, Kim YS, Lee Y, Choi IG, Kwon HJ: Expression of human beta-defensin-2 gene induced by CpG-DNA in human B cells. Biochem Biophys Res Commun 2009, 389:443-8.

\section{doi:10.1186/1471-2180-12-246}

Cite this article as: García-Pérez et al: Macropinocytosis is responsible for the uptake of pathogenic and non-pathogenic mycobacteria by $B$ lymphocytes (Raji cells). BMC Microbiology 2012 12:246.

\section{Submit your next manuscript to BioMed Central and take full advantage of:}

- Convenient online submission

- Thorough peer review

- No space constraints or color figure charges

- Immediate publication on acceptance

- Inclusion in PubMed, CAS, Scopus and Google Scholar

- Research which is freely available for redistribution 